\title{
Bioactive Materials Opportunity and Challenges in Dentistry
}

\author{
Luis Alonso Calatrava Oramas* \\ Doctor in dentistry, Universidad Central de Venezuela, Venezuela
}

*Corresponding author: Luis Alonso Calatrava Oramas, Doctor in dentistry, Universidad Central de Venezuela, Venezuela

\section{Introduction}

The global dental materials market is projected to be close to $\$ 10$ billion by 2025 [1]. Advances in dentistry are closely related to this scenario, and the primary impetus is to facilitate the workflow of dentists and increase the comfort of the patients. Therefore, notable research projects have been carried out which are currently underway, to develop new elements with improved properties, or which can be processed using advanced technologies [2-4]. The ideal material to restore or replace lost oral tissues may be difficult to achieve, but the effort persists at a determined rate. The literature is profuse with new biocompatible approaches, aimed at clinical efficiency and effectiveness; the examples in regenerative medicine, which stimulate biomimetic processes, are of great interest, but with difficulties of predictability in the clinical setting [5]. This knowledge has provided guidance for the development of biomaterials that are bioactive rather than simply biocompatible, containing additives at the nano-scale, essential components, or additional molecules and ions that have antimicrobial, tissue anabolic, or demineralizing potential [6-7].

Parallel to these advances, the compositions and properties of the composite resins have improved substantially, and it is possible to identify "development cycles". In its early days (1980-1990), the focus was on filler systems that allowed for materials with superior mechanical properties, wear resistance, and good polishing. Efforts were then directed to reduce polymerization shrinkage as a strategy to reduce postoperative sensitivity, cusp deflection, and microleakage [8]. In the current decade, bulk-fill materials are becoming increasingly popular due to the clinical appeal of depth of cure and reducing the time required to insert composite into cavity preparation $[9,10]$. In general, these current composite resins have a longer longevity, better preservation of the dental structure, adhesion to enamel and dentin surfaces, aesthetics, and proper handling. However, recurrent caries remains a predominant reason for failure, and replacement of these restorations by bacterial micro leakage throughout the composite resin/tooth reconstruction, contributes to postoperative sensitivity, inflammation, and pulp necrosis [11].

Furthermore, it has been stated that there is a potential impact of composite resins on the ecology of microorganisms in the dental bio film, due to the greater accumulation, in these restorative materials [12]. Also, the effect of the biodegradation by-products derived from them; on the important physiological functions of S. mutans indicate its potential influence on bio film formation and microbial survival on the surfaces of the oral cavity $[13,14]$.

Therefore, there is strong in vitro evidence of the synergistic effect of cyclic loading and exposure to bacteria, which aids in the penetration of the bacterial bio film at the margin of these restorations and dentin, possibly leading to development fastest secondary caries in vivo. To overcome these problems, efforts have been devoted to the development of a new generation of dental materials called by some "bioactive" that contain additives that have remineralizing and antimicrobial capabilities [15-24].

\section{Bioactive Materials in The Current Dental Market and Its Mechanism of Action [25]}

1. Materials that remineralizer, only remineralizer.

2. Materials that deposit hydroxyapatite and also remineralize.

3. Materials that stimulate pulp regeneration remineralize and deposit hydroxyapatite.

\section{Materials that Remineralize}

Fluoride is retained intraorally after treatments such as fluoridated toothpaste and the application of fluoride varnish and is then released into saliva over time. It can remain on 
teeth, mucosa, dental plaque or within a bioactive restorative. Its retention is clinically beneficial as it can be released during cariogenic challenges to decrease demineralization and improve remineralization. Materials that deposit hydroxyapatite and also remineralize. Ionomeric glasses create a new ion-enriched layer at the glass/tooth ionomer interface. This layer contains phosphate and calcium ions from dental tissues, and calcium (or strontium), phosphate and aluminum from glass ionomer cement. The remineralization process creates a harder dentin surface. The restorative fracture is usually cohesive, leaving the ion exchange layer firmly attached to the cavity wall. The dentinal tubules are sealed and protected from bacterial penetration.

There are a variety of glass ionomer derivatives: resin-modified glass ionomers, compomers, and giomers.

Giomers, represent the hybridization of the properties of glass ionomers and composite resins: the release of fluoride and the recharge of glass ionomers and the aesthetics, physical properties and handling of composite resins. Creating fluorine reservoirs releases and recharges fluorine efficiently, although not as well as glass ionomers [26].

Cention $\mathrm{N}$ is a self-curing UDMA-based direct restorative esthetic material with optional additional light-cure. The liquid is made up of dimethacrylates and initiators, while the powder contains various glass fillers, initiators and pigments. It exhibits a high density of polymer network and a high degree of polymerization throughout the depth of the restoration. Includes a special patented filler (Isofiller) that acts as a shrinkage stress reducer leading to less microleakage $[27,28]$. Among the non-resin based hydroxyapatite depositing materials, they bind with an acid-base reaction and produce an alkaline $\mathrm{pH}$ after setting. High $\mathrm{pH}$ levels (7.5 or more) seem to stimulate a more active and complete bioactivity. Ceramir (Doxa Dental, Uppsala, Sweden) is a calcium aluminate material developed for cementation that can occlude artificial marginal gaps in vitro. It works on the principle of two cements: calcium aluminate and glass ionomer cement [29]. Materials that stimulate pulp regeneration rematerialize and deposit hydroxyapatite. Bio Aggregate (Verio Dental Co. Ltd., Vancouver, Canada) is composed of nanoparticle tricalcium silicate, tantalum oxide, calcium phosphate, silicon dioxide and has improved performance compared to MTA [30].

Biodentine formulated using MTA technology, improving its physical and handling properties, with significant repairing qualities. It is a material, made of tricalcium silicate cement, zirconium oxide and calcium carbonate. The liquid is water, calcium chloride, and a water-based polymer. Which can create a dense dentin barrier and scarring of pulp fibroblasts [31]. The Theracal is also a Calcium Silicate, but modified with resin; the setting reaction of the polymerizable component is activated by light. It has been concluded that future studies should examine whether the lower calcium ion releasing capacity, together with the cytotoxic effect due to the non-polymerized resin monomers of TheraCal LC, influences its biological and clinical performance [32,33].

\section{Conclusion}

The recent research studies on the physical, chemical, and biological properties of some bioactive materials are discussed in this short review. Calcium silicate cements properties have led to a growing series of innovative clinical applications. The capacity to promote calcium-phosphate deposit suggests their use for dentin remineralization and tissue regeneration. They can induce the chemical formation of a calcium phosphate/apatite coating when immersed in biological fluids. Studies of the next generation of bioactive dental materials for "Regenerative Dentistry" will guide their clinical evolution.

\section{References}

1. https://www.marketwatch.com/press-release/global-dental-materialmarket-2019-market-growth-size-demand-trends-insights-andforecast-2025-2019-06-04.

2. Fernando D, Attik N, Pradelle-Plasse N, Jackson P, Grosgogeat B, et al. (2017) Bioactive glass for dentin remineralization: A systematic review. Mater Sci Eng C Mater Biol Appl 76(1): 1369-1377.

3. Toledano M, Cabello I, Osorio E, Aguilera FS, Medina-Castillo A, et al. (2019) Zn-containing polymer nanogels promote cervical dentin remineralization. Clin Oral Investig 23(3): 1197-1208.

4. Zhou W, Liu S, Zhou X, Hannig M, Rupf S, et al. (2019) Modifying Adhesive Materials to Improve the Longevity of Resinous Restorations. Int J Mol Scince 20(3): E723.

5. Ferracane J, Giannobile W (2014) Novel Biomaterials and Technologies for the Dental, Oral, and Craniofacial Structures. J Dent Res 93(12): 1185-1186.

6. Fernando D, Attik N, Pradelle-Plasse N, Jackson P, Grosgogeat B, et al. (2017) Bioactive glass for dentin remineralization: A systematic review. Mater Sci Eng C Mater Biol Appl 76: 1369-1377.

7. Arandi NZ, Rabi T (2018) TheraCal LC: From Biochemical and Bioactive Properties to Clinical Applications. Int J Dent 2018: 3484653.

8. Braga RR, Ferracane JL (2004) Alternatives in polymerization contraction stress management. Crit Rev Oral Biol Med 15(3): 176-184.

9. Rizzante FAP, Duque JA, Duarte MAH, Mondelli RFL, Mendonça G, et al. (2019) Polymerization shrinkage, microhardness and depth of cure of bulk fill resin composites. Dent Mater J 38(3): 403-410.

10. Leticia Cristina Cidreira Boaro, Diana Pereira Lopes, Andréia Santos Caetano de Souza, ElleaLie Nakano, Mirko DennysAyala Perez, et al. (2019) Clinical performance and chemical-physical properties of bulk fill composites resin-a systematic review and meta-analysis. Dental Materials 35(10): 249-264.

11. Demarco FF, Collares K, Correa MB, Cenci MS, Moraes RR, et al. (2017) Should my composite restorations last forever? Why are they failing? Braz Oral Res 31(suppl 1): e56.

12. Khvostenko D, Salehi S, Naleway SE, Hilton TJ, Ferracane JL (2015) Cyclic mechanical loading promotes bacterial penetration along composite restoration marginal gaps. Dent Mater 31(6): 702-710.

13. Ferracane JL (2017) Models of Caries Formation around Dental Composite Restorations. J Dent Res 96(4): 364-371. 
14. Lowe R (2017) Focus On: Bioactive Dental Materials Dentistry Today May.

15. Tiskaya M, Al-Eesa NA, Wong FSL, Hill RG (2019) Characterization of the bioactivity of two commercial composites. Dent Mater 35(12): 1757 1768.

16. Abid Althaqaf K, Sattetrhwhite J, Nikolaos Silikas (2020) A review and current state of autonomic self-healing microcapsules-based dental resin composites. Dental Materials 36(3): 329-342.

17. Kunneng Liang, Suping Wang, Siying Tao, Shimeng Xiao, Han Zhou, et al. (2019) Dental remineralization via poly(amido amine) and restorative materials containing calcium phosphate nanoparticles. Int J Oral Sci 11(2): 1-15.

18. Zhou W, Liu S, Zhou X, Hannig M, Rupf S, et al. (2019) Modifying Adhesive Materials to Improve the Longevity of Resinous Restorations. Int J Mol Sci 20(3): E723.

19. Goldstep F (2017) Bioactivity In Restorative Dentistry: A User's Guide Oral health 1-15.

20. Gordan VV, Blaser PK, Watson RE, Mjör IA, McEdward DL, et al. (2014) A clinical evaluation of a giomer restorative system containing surface prereacted glass ionomer filler: results from a 13-year recall examination. J Am Dent Assoc 145(10): 1036-1043.

21. Acharya RP, Morgano SM, Luke AC, Ehrenberg D, Weiner S, et al. (2018) Retentive strength and marginal discrepancies of a ceramic-reinforced calcium phosphate luting agent: An in vitro pilot study. J Prosthet Dent 120(5):771-779.

22. Arandi NZ, Rabi T (2018) TheraCal LC: From Biochemical and Bioactive Properties to Clinical Applications. Int J Dent 2018: 3484653.

23. Lin Lu Dai, May Lei Mei, Chun Hung Chu, Edward Chin Man Lo (2019) Mechanisms of Bioactive Glass on Caries Management: A Review. Materials (Basel) 12(24): 4183.
24. Md. Minhajul Islam, Md. Shahruzzaman, Shanta Biswas, Md. Nurus Sakib, Taslim U Rashid, et al. (2020) Chitosan based bioactive materials in tissue engineering applications-A review. Bioact Mater 5(1): 164-183.

25. Goldstep F (2018) Bioactivity In Restorative Dentistry: A User's Guide Oral health.

26. Rusnac ME, Gasparik C, Irimie AI, Grațian Grecu, A Mesaroș, et al. (2019) Giomers in dentistry-at the boundary between dental composites and glass-ionomers. Med Pharm Rep 92(2): 123-128.

27. Manpreet Kaur, Navjot Singh Mann, Ashu Jhamb, Dr. Divya Batra A (2019) comparative evaluation of compressive strength of Cention N with glass Ionomer cement: An in-vitro study. International Journal of Applied Dental Sciences 5(1): 05-09.

28. Tiskaya M, Al-Eesa NA, Wong FSL, Hill RG (2019) Characterization of the bioactivity of two commercial composites. Dent Mater 35(12):17571768.

29. Engstrand J, Unosson E, Engqvist H (2012) Hydroxyapatite Formation on a Novel Dental Cement in Human Saliva. ISRN Dent 2012: 624056.

30. Jia Kim, Young-Sang Song, Kyung-San Min, Sun-Hun Kim, JeongTae Koh, et al. (2016) Evaluation of reparative dentin formation of ProRoot MTA, Biodentine and BioAggregate using micro-CT and immunohistochemistry. Restor Dent Endod 41(1): 29-36.

31. Lipski M, Nowicka A, Kot K, Postek-Stefańska L, WysoczańskaJankowicz,I, et al. (2018) Factors affecting the outcomes of direct pulp capping using Biodentine. Clin Oral Investig 22(5): 2021-2029.

32.Ziad Arandi, N Rabi T (2018) TheraCal LC: From Biochemical and Bioactive Properties to Clinical Applications Int J Dent 2018: 3484653.

33. Young Kyung Kim, Mi-Hee Hong, Tae-Yub Kwon (2020) Dentin Bonding of TheraCal LC Calcium Silicate Containing an Acidic Monomer: An In Vitro Study. Materials (Basel) 13(2): 293.

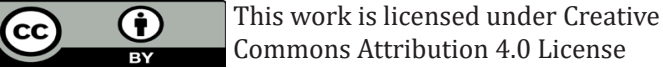

To Submit Your Article Click Here:

Submit Article

DOI: $10.32474 /$ RRHOAJ.2020.05.000208

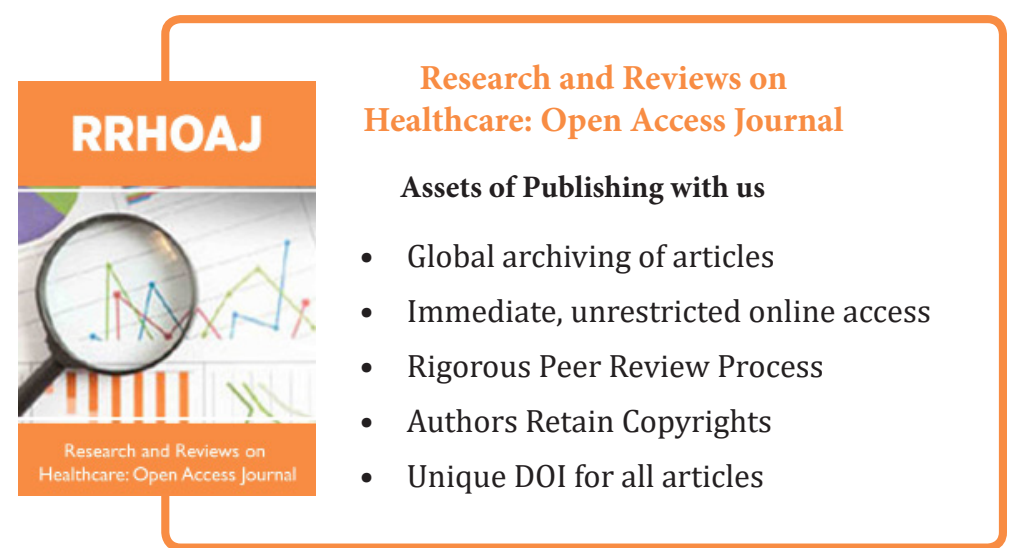

Citation: Luis Alonso Calatrava Oramas. Bioactive Materials Opportunity and Challenges in Dentistry. Res \& Rev Health Care Open Acc J 